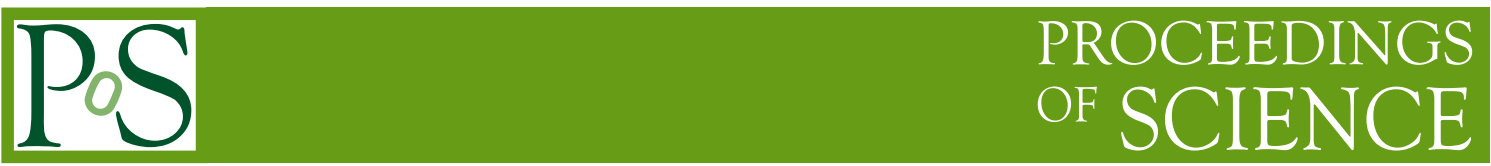

\title{
Heavy resonance searches at the FCC-hh
}

\author{
Clement Helsens* \\ CERN, \\ E-mail: clement.helsens@cern.ch
}

\begin{abstract}
The physics reach and feasibility of the Future Circular Colliders (FCC) are currently being investigated in the form of Conceptual Design Reports. The ultimate goal of FCC is to collide protons with a centre-of-mass energies of $100 \mathrm{TeV}$, thus extending the reach of the current HEP facilities. This high-energy regime opens new opportunities for the discovery of physics beyond the Standard Model (SM), but also for precise measurement of fundamental parameters of the SM. Following the LEP/LHC model, it is obvious that the first collider that should be operated, is an electron-positron machine, FCC-ee. It aims at collecting multi-ab ${ }^{-1}$ integrated luminosities in $e^{+} e^{-}$collisions at different center-of-mass energies, yielding $10^{12} \mathrm{Z}$ bosons, $10^{8} \mathrm{~W}^{+} \mathrm{W}^{-}$pairs, $10^{6}$ Higgs bosons and $10^{6} t \bar{t}$ pairs. In these proceedings we explore the physics reach of the proton-proton Future Circular Collider (FCC-hh) for searches of new particles decaying to two high energetic leptons $l^{+} l^{-}(l=e, \mu, \tau)$, jets (non-tops), tops and W/Z boson. We discuss the expected exclusion limits and discovery potential for benchmark models predicting new massive particles that result in resonant structures in the invariant mass spectrum. The study is based on large statistic samples of generated events processed through a parametric simulation of the expected performance of an FCC-hh detector. The center-of-mass energy of $\sqrt{s}=100 \mathrm{TeV}$ makes it the ultimate machine for such new heavy particles, and studying their characteristics is also extremely relevant to discuss the main limitations of the detector to precisely discriminate high energetic top-quarks or W/Z bosons from standard QCD-jets.
\end{abstract}

The 39th International Conference on High Energy Physics (ICHEP2018)

4-11 July, 2018

Seoul, Korea

${ }^{*}$ Speaker. 


\section{Introduction}

The design of a $100 \mathrm{TeV}$ proton-proton collider leads to many challenges for detector design. Detailed optimisation's of the detector is needed in order to achieve the required physics goals. The capabilities of such a detector should include the capacity of measuring multi-TeV leptons, top-quarks and bosons. The goal of the study presented in this document is to categorise the discovery reach for heavy objects and find ways to discriminate multi-jets from top and boson-jets. Monte Carlo (MC) simulated event samples were used to simulate the response of the FCC-hh detector to signal and backgrounds. Signals are generated with Pythia 8.230 [1] using the leading order cross-section from the generator. The Standard Model (SM) backgrounds are DrellYan, di-jet (QCD), top pairs $(t \bar{t}), V V$ and $V+$ jets where $V=W / Z$, which were generated using MG5_aMC@NLO 2.5.2 [2] at leading order only. A k-factor of 2 is applied to all the background processes to account for higher order corrections. The $\mathrm{MC}$ events are then processed trough a parametric simulation (Delphes [3]) of the expected performance of an FCC-hh detector [4] integrated inside the full FCC software stack. For the studies summarised in this document, no pile-up is assumed, but for such heavy object the effect it is expected to have a small impact. The assumptions made in terms of detector performance are being validated in full simulation. For all the results presented below, hypothesis testing is performed using a modified frequentist method based on a profile likelihood that takes into account the systematic uncertainties as nuisance parameters that are fitted to the expected Monte-Carlo. The results will be summarised for three different integrated luminosity scenarios: 1) 10 years at baseline instantaneous luminosity representing $2.5 a b^{-1}$ 2), adding 15 years at ultimate for a total of $30 a b^{-1}, 3$ ) and as an extreme point $100 a b^{-1}$.

\section{Resonances decaying to leptons}

Models with extended gauge groups often feature additional U(1) symmetries with corresponding heavy spin- 1 bosons. These bosons, generally referred to as $Z^{\prime}$, would manifest themselves as a narrow resonance in the dilepton mass spectrum. Among these models are those inspired by Grand Unified Theories (GUT), motivated by gauge unification or a restoration of the left-right symmetry violated by the weak interaction. Examples include the $Z^{\prime}$ bosons of the E6 motivated theories [5, 6] and Minimal models [7]. The Sequential Standard Model (SSM) [9] posits a $Z_{\text {SSM }}^{\prime}$ boson with couplings to fermions that are identical to those of the Standard Model $Z$ boson. The decay products of heavy resonances are in the multi- $\mathrm{TeV}$ regime and the capability to reconstruct their momentum imposes stringent requirement on the detector design. In particular, reconstructing the track curvature of multi-TeV muons requires excellent position resolution and a large lever arm. In this section, the expected sensitivity is presented for a $Z^{\prime} \rightarrow \ell \ell$ (where $\ell=\mathrm{e}, \mu$ ) and $Z^{\prime} \rightarrow \tau \tau$ separately. All lepton flavour decays of the $Z^{\prime}$ are generated assuming universality of the couplings.

Events are required to contain two leptons with $p_{T}>1 \mathrm{TeV},|\eta|<4$ and an invariant mass $M_{l l}>2.5 \mathrm{TeV}$. Figure 1 left shows the di-muon invariant mass for an hypothetical 30/,TeV signal. For di- $\tau$ final state we focus on the most sensitive fully hadronic channel only. The di- $\tau$ event selection requires two jets with $p_{T}>0.5 \mathrm{TeV}$ and $|\eta|<2.5$ identified as $\tau$ 's. To ensure no overlap between the $\ell$ and $\tau$ final states, jets containing leptons with $p_{T}>100 \mathrm{GeV}$ are vetoed. Finally, requirements of $\Delta \phi\left(\tau_{1}, \tau_{2}\right)>2$ and $2.5<\Delta R\left(\tau_{1}, \tau_{2}\right)<4$ are applied. Mass dependent cuts are applied 
to maximise the signal to background ratio for the $Z^{\prime} \rightarrow \tau \tau$ channel. For the $e e$ and $\mu \mu$ analyses, the di-lepton invariant mass is used as discriminant, while for the $\tau \tau$ channel the transverse mass ${ }^{1}$ is used. A 50\% uncertainty on the background normalisation is assumed. The very large centre of mass energy provides a correspondingly large mass reach. For the $e e$ and $\mu \mu$ cases masses up to $42 \mathrm{TeV}$ can be excluded or discovered. Heavy resonance decaying to $\tau$ leptons reconstructed in the hadronic decay mode are more challenging, because of the overwhelming di-jet background, but massses up to $18 \mathrm{TeV}$ could be probed.

\section{Hadronic resonances}

Many models of beyond the SM (BSM) physics predict additional particles with masses at the TeV scale. The presence of new resonant states [8] decaying to two highly boosted particles decaying hadronically could be observed as an excess in the QCD dijet distribution. We focus here on three specific benchmark models: a $Z_{\text {SSM }}^{\prime}$ [9], a Randall-Sundrum graviton [8], and an excited quark resonance [10]. We study the sensitivity in the following benchmarks using hadronic decay modes of $Z^{\prime} \rightarrow t \bar{t}, G_{R S} \rightarrow W W$ and $Q^{*} \rightarrow j j$. The decay products are typically in the multi-TeV regime and their reconstruction imposes stringent requirement on the detector design. Precise jet energy resolution requires full longitudinal shower containment. Highly boosted top quarks and $W$ bosons decay into highly collimated jets that need to be disentangled from standard QCD jets by studying their substructure. High discrimination power and sensitivity for these searches at such extreme energies, requires excellent granularity both in the tracking detectors and calorimeters.

For the $Q^{*} \rightarrow j j$ analysis, jets are clustered using Delphes particle-flow candidates with the anti- $k_{T}$ algorithm with parameter $\mathrm{R}=0.4$. At least two jets with $p_{T}>3 \mathrm{TeV}$ and $|\eta<3|$ are required and the rapidity difference between the two leading jets to be smaller, $\Delta(\eta)<1.5$ as di-jet events will tend to be more central.

For the $Z^{\prime} \rightarrow t \bar{t}, G_{R S} \rightarrow W W$ analyses, as it has been found that Delphes track jets are better able to resolve the jet sub-structure compared to particle-flow jets, therefore the searches are performed using track jets. As no lepton veto is applied, there is also some acceptance for leptonic decays of the $W$. The sensitivity to semi-leptonic $W W$ or $t \bar{t}$ decays is enhanced by adding the $\overrightarrow{p_{T}}$ miss vector to the closest jet 4-momentum (among the to leading jets). We require two jets with a $p_{T}>3 \mathrm{TeV}$ and $|\eta<3|$ and $\Delta(\eta)<2.4$. Both jets must either be $W$ or top tagged by requiring a high multivariate score from a specially developed tagger based on boosted decision trees and designed to discriminate QCD-jets versus $W$ and top jets. Both high- $p_{T}$ jets must be $b$-tagged for the $Z^{\prime} \rightarrow t \bar{t}$ analysis. Finally, to further reject QCD, we require for both jets a cut on the softdropped mass $m_{S D}>40 \mathrm{GeV}$.

For the $Q^{*} \rightarrow j j$ masses up to $40 \mathrm{TeV}$ could be discovered with $30 a b^{-1}$. Reconstructing heavy resonances decaying to $W W$ and $t \bar{t}$ is more challenging and requires the use of novel approaches to boosted object tagging to reduce the backgrounds. The reach for $Z^{\prime} \rightarrow t \bar{t}$ (in TC2 models) and $G_{R S} \rightarrow W W$ is $24 \mathrm{TeV}$ and $22 \mathrm{TeV}$ respectively and it is possible to discover a $Z_{S S M}^{\prime} \rightarrow t \bar{t}$ up to $m_{Z}=18 \mathrm{TeV}$.

\footnotetext{
${ }^{1}$ the transverse mass is defined as $m_{T}=\sqrt{2 * E_{T}^{\text {miss }} *\left(1-\cos \Delta \phi\left(Z^{\prime}, E_{T}^{\text {miss }}\right)\right)}$
} 

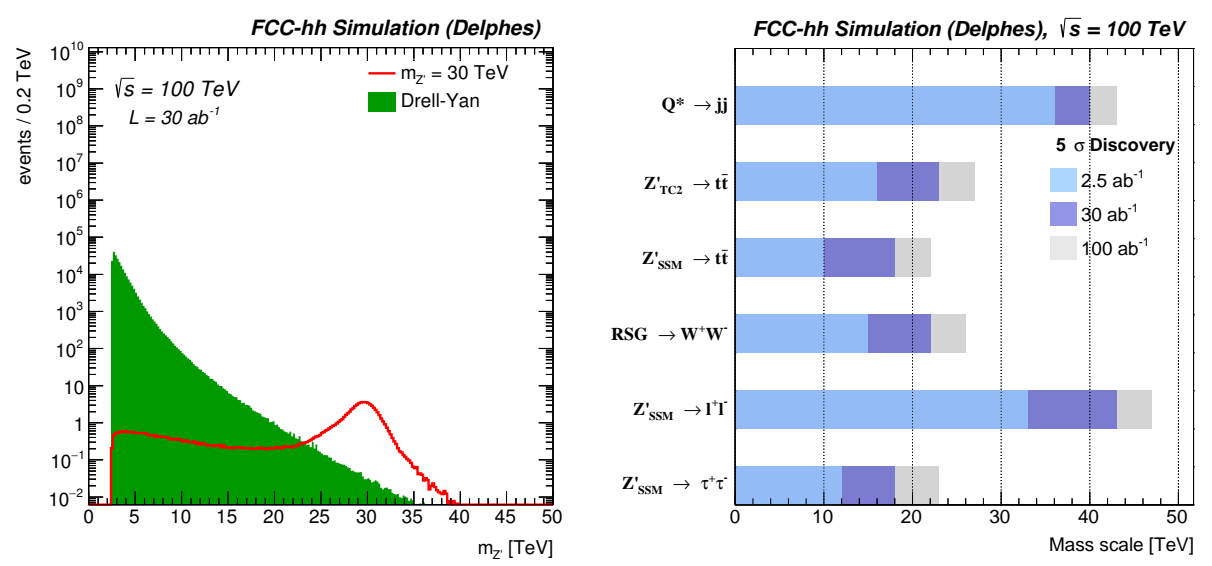

Figure 1: Left: di-muon invariant mass for an hypothetical $30 \mathrm{TeV} Z_{\mathrm{SSM}}^{\prime}$ considering $30 a b^{-1}$ of integrated luminosity. Right discovery reach for heavy resonances in the different assumptions in the text.

\section{Summary}

While the LHC do not give clear guidance of which BSM scenario and mass scale to look for, rather general arguments support $100 \mathrm{TeV}$ as a sensible target for FCC-hh. There is evidence that $100 \mathrm{TeV}$ is necessary and sufficient to achieve crucial measurements and give clear yes/no answers to some of the important questions that might still be left open after the HL-LHC and to explore scenarios that could emerge from the FCC-ee phase. Concerning the direct search for heavy resonances at FCC-hh the results are summarised in figure 1 right for different integrated luminosity scenarios.

\section{References}

[1] T. Sjöstrand et al., An Introduction to PYTHIA 8.2, Comput. Phys. Commun. 191 (2015) 159

[2] J. Alwall et al., The automated computation of tree-level and next-to-leading order differential cross sections, and their matching to parton shower simulations, JHEP 1407 (2014) 079

[3] J. de Favereau et al., DELPHES 3, A modular framework for fast simulation of a generic collider experiment, JHEP 1402 (2014) 057 [arXiv:1307.6346 [hep-ex]].

[4] FCC Design Reports - Contributor Registration https://indico.cern.ch/event/750953/overview

[5] D. London and J. L. Rosner, Extra Gauge Bosons in E(6),

[6] A. Joglekar and J. L. Rosner, Searching for signatures of $E_{6}$, Phys. Rev. D 96 (2017) no.1, 015026

[7] E. Salvioni, G. Villadoro and F. Zwirner, Minimal Z-prime models: Present bounds and early LHC reach, JHEP 0911 (2009) 068 [arXiv:0909.1320 [hep-ph]].

[8] L. Randall and R. Sundrum, A Large mass hierarchy from a small extra dimension, Phys. Rev. Lett. 83 (1999) 3370 [hep-ph/9905221].

[9] P. Langacker, The Physics of Heavy Z' Gauge Bosons, Rev. Mod. Phys. 81 (2009) 1199

[10] U. Baur, M. Spira and P. M. Zerwas, Excited Quark and Lepton Production at Hadron Colliders, Phys. Rev. D 42 (1990) 815. 